\title{
LOW-DOSE CBCT FOR LOCALIZATION OF IMPACTED SUPERNUMERARY TEETH IN CHILDREN
}

\author{
Fatma Abdelgawad", Ahmed Abd Alsamad"* and Nada Mohamed Wassef*
}

\begin{abstract}
Background: In children, impacted supernumerary teeth usually present problems as delayed eruption of the permanent successors, leading to esthetics and functional problems. The accurate localization of these impacted supernumerary teeth is mandatory to avoid injuring the developing permanent teeth during surgical removal. Use of low-dose CBCT can provide a detailed threedimensional image facilitating its use in children.
\end{abstract}

Aim: The aim of this study is to determine the accuracy of low-dose CBCT in localization of impacted supernumerary teeth before surgical removal in children.

Methods: Ten children having unerupted permanent incisors were recruited. Periapical radiographs were done to confirm the presence of impacted supernumerary teeth. A lowdose CBCT was done for each child with proper interpretation and measurements obtained for accurate localization. Surgery was done after that for all cases to confirm the proper location and measurements gathered from the cone beam interpretation using a periodontal probe.

Results: With the aid of the measures obtained from the interpretation of the low-dose CBCT, there was an increased accuracy in the surgical approach for removing the supernumerary teeth. In addition, there was a reduction in the number of radiographs needed for accurate localization and decreased tissue injury and possibility of damage for the permanent successors or adjacent teeth.

Conclusion/Clinical Significance: Low-dose CBCT can be used for better evaluation and localization of impacted supernumerary teeth before surgery.

KEYWORDS: Low-dose CBCT, supernumerary teeth, mesiodens, cone beam computed tomography.

\section{INTRODUCTION}

Supernumerary teeth are teeth present in excess to the normal number of teeth and may be present in different areas in the mouth, with the maxillary anterior region considered the most common area (Omami et al., 2015). Their number may vary from one to multiple teeth and may appear unilaterally or bilaterally. In some cases, these teeth may erupt

* Lecturer of Pediatric Dentistry and Dental Public Health, Faculty of Dentistry, Cairo University, Egypt.

** Associate professor of Oral and Maxillofacial Radiology, Faculty of Dentistry, Cairo University, Egypt. 
or remain impacted either in an upright position or inverted (Ziegler \& Klimowicz, 2013). The shape of supernumerary teeth varies from an odontome to supplemental teeth. Mesiodens are in the midline between the central incisors, whether erupted or not (Gurler at al., 2017). The prevalence of supernumerary teeth is variable and may range between 0.1 and $3.8 \%$ and is more common in the permanent dentition (Rajab \& Hamdan, 2002 and Ziegler \& Klimowicz, 2013).

Supernumerary teeth may remain asymptomatic for years and may be discovered accidentally. However, some complications may arise due to impacted supernumerary teeth including delay in permanent teeth eruption, deviation in the path of eruption, adjacent teeth displacement, root resorption, dentigerous cysts, in addition to the esthetic and functional impacts on the children due to the delayed eruption of the successors (Parolia et al., 2011 and Omami et al., 2015).

Surgical removal of supernumerary teeth is sometimes mandatory, and in case of young age with mixed dentition, care must be taken to avoid any damage or harm to the permanent successors or important structures as blood vessel and nerves in the field of surgery (AAPD, 2015).

Accurate localization of the teeth and estimation of their proximity to important structures can be obtained by using conventional radiographs to reach a 3D visualization of the operation field. Panoramic radiograph in addition to periapical radiographs with tube-shift technique (Jeremias et al., 2016) or radiographs at right angle to one another or occlusal radiographs can be used. The introduction of the cone beam computed tomography can aid in accurate localization for precise surgical procedures (Katheria et al., 2010 and AAPD, 2015).

The use of low-dose CBCT can be of great benefit in children, where there is a reduced dose of radiation while the accuracy of the images for localization is highly precise (Hidalgo Rivas et al., 2015).
Therefore, the aim of this study is to determine the accuracy of low-dose CBCT in localization of impacted supernumerary teeth before surgical removal in children.

\section{SUBJECTS AND METHODS}

\section{Subjects:}

Approval from Research Ethics committee (REC), Faculty of Dentistry, Cairo University was obtained. Children were selected from the outpatient clinic in Pediatric Dentistry and Dental Public Health Department, Faculty of Dentistry, Cairo University complaining of unerupted permanent anterior teeth. Routine periapical radiographs were performed to confirm the presence or absence of any supernumerary teeth that might prevent the eruption of permanent teeth Fig. (1). After confirming the presence of the supernumerary teeth, a written consent to participate in the study was obtained from the parents.

\section{Methods:}

\section{A-Low-dose CBCT:}

The patients were referred to the outpatient clinic of Oral and Maxillofacial Radiology Department, Faculty of Dentistry, Cairo University for CBCT imaging using Planmeca ProMax® 3D Mid (Planmeca, Helsinki, Finland).

The patient was positioned on the CBCT machine where the positioning laser beam of the machine was used to seat the patient so that the mid-sagittal plane and the occlusal plane were perpendicular and horizontal to the floor, respectively. The used parameters in the low-dose protocol were $90 \mathrm{kVp}$ $5 \mathrm{~mA}-2.8$ seconds with field of view $40 \times 50 \mathrm{~mm}$ (which included the anterior teeth of the maxilla) and voxel size $0.4 \mathrm{~mm}$ (low dose CBCT). The resultant $\mathrm{CBCT}$ images (3D image, cross sectional cuts as well as Reformatted panoramic image) were assessed using Planmeca Romexis ${ }^{\circledR}$ 3.5.1. software 


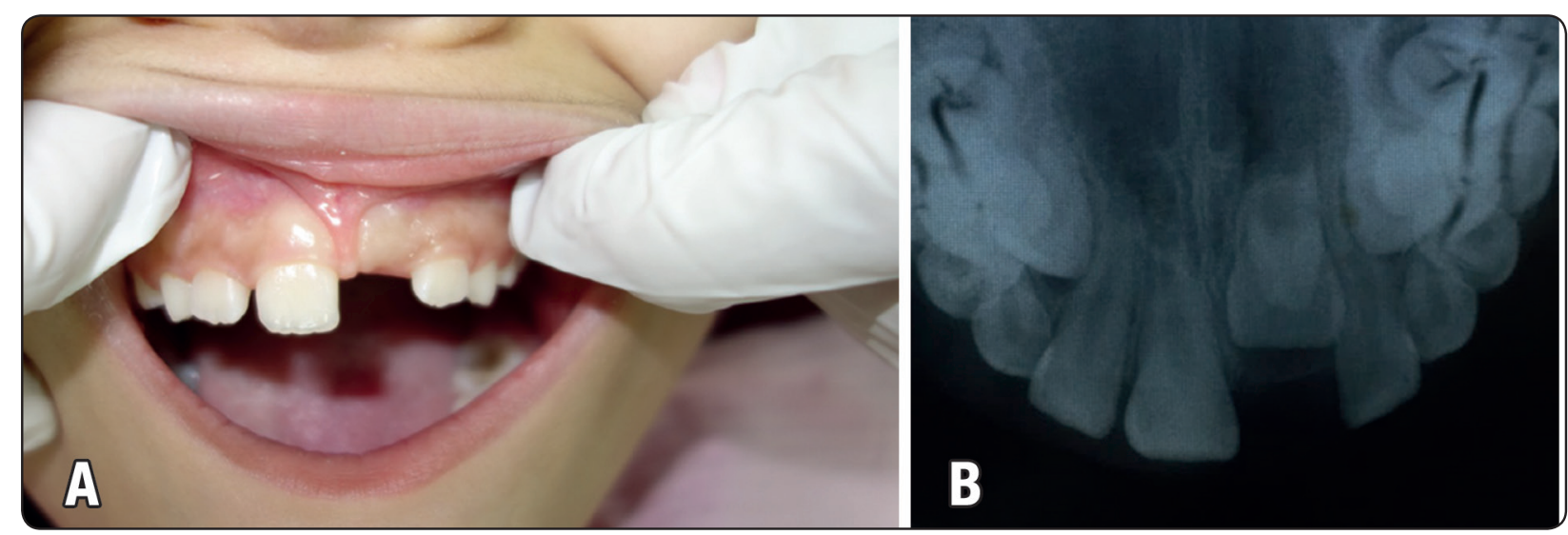

Fig. (1): (A) Pre-operative photograph showing unerupted permanent central incisor and (B) periapical radiograph showing unerupted central incisor and supernumerary tooth.

(Planmeca, Helsinki, Finland) to accurately locate the impacted supernumerary teeth and determine whether they are labial or palatal and inverted or not and to obtain a 3D visualization Fig. (2).

The images were studied thoroughly, and measurements were estimated using the CBCT images to plan the surgical procedure and estimate the needed steps to minimize tissue trauma and unnecessary manipulation in the bone and tissues Fig. (3).

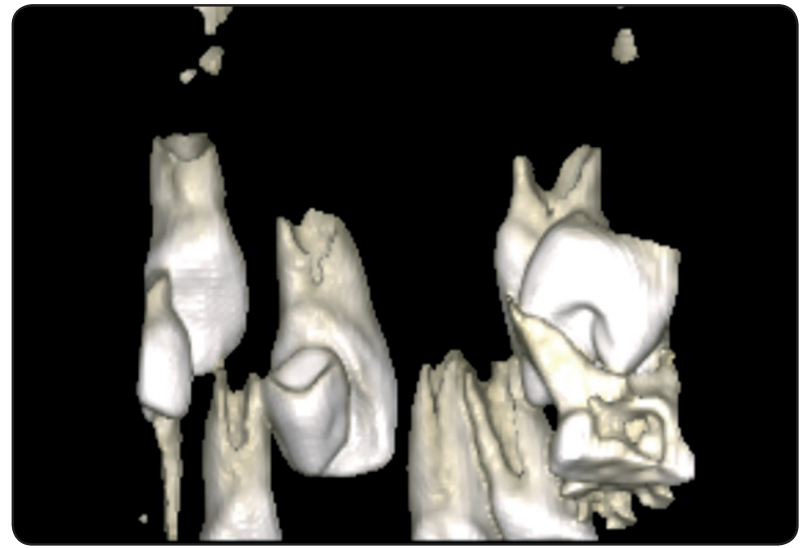

Fig. (2): Palatal view of the 3D image regenerated from the low-dose CBCT, showing palatally positioned supernumerary tooth.

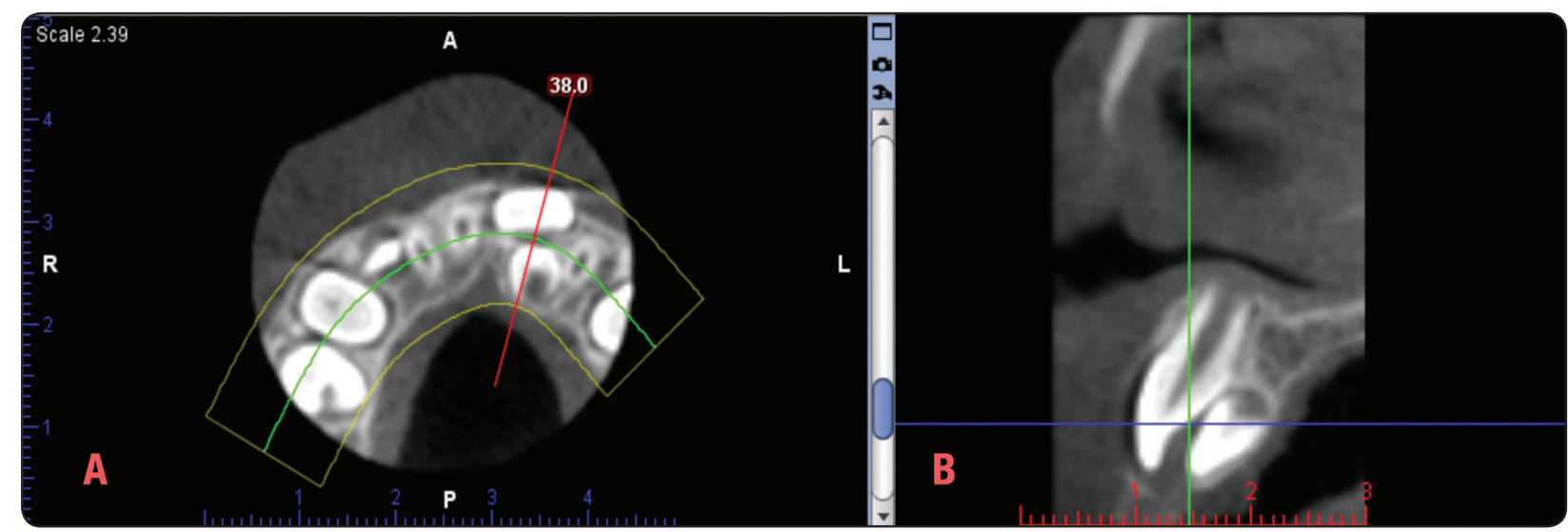

Fig. (3): CBCT images used to accurately locate the exact position of the supernumerary tooth (A) Occlusal view and (B) Sagittal view. 


\section{B-Surgical Procedures}

For all patients topical anesthesia was applied using flavored $20 \%$ benzocaine topical anesthesia gel (Iolite gel, Dharma Research Inc., USA) for about 2 min. Labial infiltration local anesthesia was administered using articaine $4 \%$ with epinephrine 1:100,000 (Artinibsa, Inibsa, Barcelona, Spain) using a short needle (gauge 30). After effective anesthesia was obtained intrapapillary injection was used followed by palatal injection of the local anesthetic drug. Surgical incision was performed, and a full thickness palatal or labial flap was raised according to the planned location from the CBCT using a mucoperiosteal elevator. A periodontal probe was used to accurately locate the points of access to the supernumerary teeth Fig. (4). In some patients, bone removal was needed to reach the impacted tooth. After gaining access to the supernumerary tooth, the tooth was luxated and removed. The flap was approximated and sutured. The patients and parents were given post-surgical instructions (soft diet, proper oral hygiene measures, and saline mouthwash). After one week, the sutures were removed.

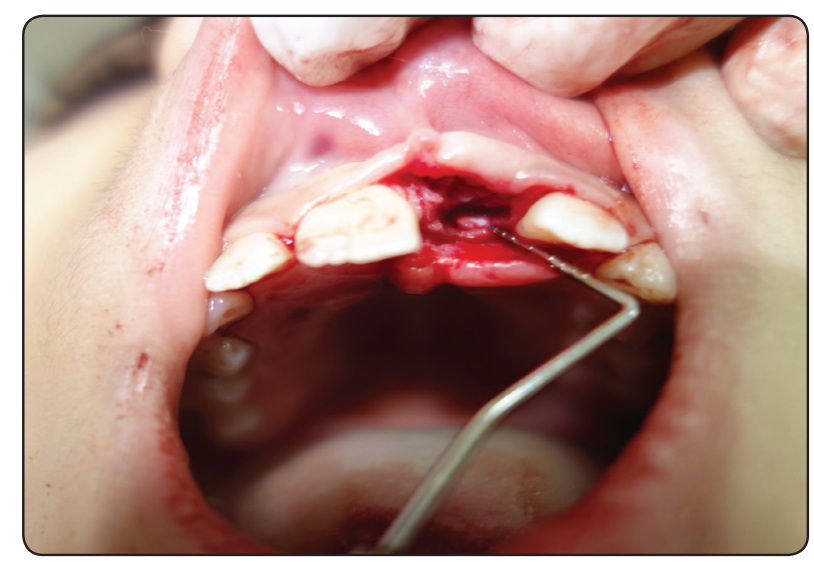

Fig. (4): Surgical operation and accurate localization of the supernumerary tooth using periodontal probe.

\section{RESULTS}

Patients enrolled in the study were 6 males and 4 females, their ages ranged between 8 12 years old with a mean $(9.5 \pm 1.42)$. A number of 15 supernumerary teeth were present in the
10 children as there were cases with one, two or three supernumerary teeth per child. One patient had three supernumerary teeth, three patients had two supernumerary teeth and six patients had one supernumerary tooth.

In all patients, the use of measurements obtained from the low-dose CBCT images aided accurate surgical steps to reach the surgical site with minimal manipulation or unnecessary cutting of tissues. There was $100 \%$ success in proper localization of the maxillary supernumerary teeth as it was planned before the surgical procedure from the interpretation of the CBCT images without any unnecessary bone removal or tissue cutting.

\section{DISCUSSION}

Children with impacted supernumerary teeth present challenges to dentists, as they are already affected esthetically and functionally by the delayed eruption of their teeth. In addition, surgical procedures in case of young age require the most accurate manipulation to avoid any damage to the successors. Therefore, in such cases 3D image regeneration is mandatory to reach the precise localization of the supernumerary teeth without any damage to the successors and the neighboring structures as nerves and blood vessels.

For 3D image generation CBCT has advantages over conventional $\mathrm{CT}$ including increased image accuracy, rapid scan time, reduced radiation dose, and reduced image artifacts (Dhillon \& Kalra, 2013). The low-dose CBCT may present advantage over conventional $\mathrm{CBCT}$ where the radiation dose is lowered without affecting the accuracy of the images that can be used for accurate clinical interpretation as agreed upon by (Hidalgo Rivas et al., 2015).

In this study, the use of low-dose CBCT helped the operators reach the precise accurate localization of the supernumerary teeth. The 3D images, in addition to the accurate measurements obtained on the computer software allowed the operators 
to minimize tissue damage during the surgical procedures, where the surgical incision sites and bone removal amounts and sites where accurately planned for the surgery. Bone removal (when indicated) was minimal as the site of the tooth was accurately estimated. These results are in accordance with the results of Schwindling et al., 2019, where they stated that using low-dose CBCT did not affect the accuracy of the measurements in addition to their advantages of lowered radiation exposure.

Regarding the patients in this study, they all came complaining of the delayed eruption of their anterior teeth, that affected their esthetics, function and in many of them their self-esteem to the extent that they avoided smiling. The use of the periodontal probe as a guide for the measurements during surgical steps was useful as it was easily used by the operator, readily available and non-expensive.

Since it is important to balance the risks and benefits of radiation exposure especially in children (Tosta et al., 2013) and considering the ALARA principle (keeping the radiation dose to the patients as low as reasonably achievable) (Okano and Sur, 2010), the use of low-dose CBCT with the used parameters $\left(52 \mathrm{mGy} \mathrm{cm}^{2}\right.$ in terms of (DAP) Dose Area Product) can be considered safe, feasible and accurate.

The use of low-dose CBCT can therefore be justified in such cases of supernumerary teeth as it is in accordance with the three basic principles of radiation protection (justification, limitation, and optimisation) stated by (Aps, 2013).

\section{CONCLUSION/CLINICAL SIGNIfiCANCE}

Low-dose CBCT can be used for better evaluation and localization of impacted supernumerary teeth before surgery.

\section{REFERENCES}

- American Academy of Pediatric Dentistry (2015) Guideline on Management Considerations for Pediatric
Oral Surgery and Oral Pathology. vol. 37. pp. 279-288.

- $\quad$ Aps, J. K. M. (2013) Cone Beam Computed Tomography in Paediatric Dentistry: Overview of Recent Literature. vol. 14. pp. 131-140.

- Dhillon, J. K. and Kalra, G. (2013) Cone Beam Computed Tomography: An Innovative Tool in Pediatric Dentistry. J Pediatric Dent, 1, pp. 27-31.

- Gurler, G., Delilbaşi, C. \& Delilbaşi, E. (2017). Investigation of Impacted Supernumerary teeth: A Cone Beam Computed Tomograph (CBCT) Study. Journal of Istanbul University Faculty of Dentistry, 51. 10.17096/ jiufd.20098.

- Hidalgo Rivas, J. A., Horner, K., Thiruvenkatachari, B., Davies, J. \& Theodorakou, C. (2015) Development of a Low-Dose Protocol for Cone Beam CT Examinations of the Anterior Maxilla in Children. British Journal of Radiology, 88 (1054) October, p. 20150559.

- Jeremias, F., Maria, C., Fragelli, B., Di, S., Mastrantonio, S., Santos-pinto, L. \& Santos-pinto, A. (2016) Cone-Beam Computed Tomography as a Surgical Guide to Impacted Anterior Teeth. Dental Research Journal, 13, pp. 85-90.

- $\quad$ Katheria, B. C., Kau, C. H., Tate, R., Chen, J.-W., English, J. \& Bouquot, J. (2010) Effectiveness of Impacted and Supernumerary Tooth Diagnosis from Traditional Radiography versus Cone Beam Computed Tomography. Pediatric dentistry, 32 (4), pp. 304-309.

- Okano, T. \& Sur, J. (2010) Radiation Dose and Protection in Dentistry. vol. 46. pp. 112-121.

- Omami, M., Chokri, A., Hentati, H. \& Selmi, J. (2015) Cone-Beam Computed Tomography Exploration and Surgical Management of Palatal, Inverted, and Impacted Mesiodens. Contemporary Clinical Dentistry, 6 (6), p. 289.

- Parolia, A., Kundabala, M., Dahal, M., Mohan, M., Thomas, M. S. (2011) Management of supernumerary teeth. J Conserv Dent, 14, pp. 221-4.

- Rajab, L. D. \& Hamdan, M. A. (2002) Supernumerary teeth: Review of the literature and a survey of 152 cases. Int J Pediatr Dent, 12, pp. 244-54.

- $\quad$ Schwindling, F. S., Hilgenfeld, T., Weber, D., Kosinski, M.A., Rammelsberg, P., Tasaka, A. (2019) In vitro Diagnostic Accuracy of Low-dose CBCT for Evaluation of Peri-implant Bone Lesions. Clin Oral Impl Res, 00, pp. $1-9$ 
- $\quad$ Tosta, M., Kawagoe, S. T., Imura, N., Souza Filho, F. J., Silva, R.C., Ferraz, C.R. (2013) CBCT-guided Endodontic Management of Maxillary Central Incisor Fused to Mesiodens: A Case Report. Dental Press Endod., 3(2), pp. $90-5$.
- Ziegler, C. M. \& Klimowicz, T. R. (2013) A Comparison between Various Radiological Techniques in the Localization and Analysis of Impacted and Supernumerary Teeth. Indian journal of dental research: official publication of Indian Society for Dental Research, 24 (3), pp. 336-341. 